\section{Fraction, Cycle, or a New Terminology? What Would Be Most Appropriate for Molecularly Targeted Radiotherapy with Unsealed Sources?}

TO THE EDITOR: Targeted radionuclide therapy with unsealed sources constitutes a unique form of therapy that encompasses the characteristics of both radiotherapy and systemic chemotherapy to a varying extent. Its administration, delivery, and localization can be viewed akin to those of a systemic pharmaceutical whereas the resultant therapeutic effect at the cellular target is primarily through the radiation dose delivered by the radiopharmaceutical. The therapeutic agent is localized at the target by a particular metabolic pathway or through cell-surface receptors-enzymes or peptides that have been probed by prior diagnostic imaging — forming the basis of theranostics, a popular term in recent years in the domain of unsealed molecularly based radionuclide therapy.

The dose schedule in chemotherapy is typically described by the term cycle (where a course consists of multiple cycles of chemotherapy with an interim rest period between cycles), whereas the individual radiotherapy doses are denoted by the term fractions. In unsealed radiopharmaceutical therapy, to emphasize the final therapeutic effect at the target (which is primarily by radiation-induced DNA damage), each individual dose was initially denoted by the term fraction. In recent years, however, cycle has been more frequently used (including in guidelines), particularly for intravenous radiopharmaceutical treatment (such as peptide receptor radionuclide therapy with ${ }^{177} \mathrm{Lu} /{ }^{90}$ Y-DOTATATE), which is usually scheduled at a multiple regular intervals, each given the term one cycle (1). One can partly conceive that this trend shift is due to the apparent similarity between the newer intravenous therapies and systemic chemotherapies, including the issues encountered and their management. Truly, current therapies such as peptide receptor radionuclide therapy have a substantial similarity to chemotherapy schedules (including complete and efficient management of associated complications such as emesis and others both during therapy and afterward, which demands that the attending nuclear medicine physician have sound medical knowledge). In day-to-day practice, however, both fraction and cycle are frequently used interchangeably.

Another perspective is the concept of dose fractionation. Dose fractionation schedules continue to evolve in systemic radionuclide therapy and, compared with external radiotherapy, are relatively less well defined at present; among many factors, the schedule is likely to be governed by the effect achieved, the intent of therapy (neoadjuvant vs. palliative), and the biology of the tumor in question (2). Interestingly, when fractionation is based on these characteristics, the fractionated doses are at times referred to as cycles administered to the patient.

With the progression of theranostics and radionuclide therapies and the introduction of several novel unsealed systemic therapeutic agents (including the introduction of $\alpha$-emitting radiopharmaceuticals) into the clinical domain, the potential of this form of therapy is likely to expand rapidly in coming years. Hence, understanding the complexities of dosimetry-related radiobiology and appropriating the associated terminology for dose schedule is a need of the hour and ought to be considered by the nuclear medicine fraternity.

\section{REFERENCES}

1. Bodei L, Mueller-Brand J, Baum RP, et al. The joint IAEA, EANM, and SNMMI practical guidance on peptide receptor radionuclide therapy (PRRNT) in neuroendocrine tumours. Eur J Nucl Med Mol Imaging. 2013;40:800-816.

2. Basu S. Dose fractionation in ${ }^{131}$ I-metaiodobenzylguanidine (MIBG) therapy: should the tumour biology and intent of therapy be the guide? Eur J Nucl Med Mol Imaging. 2010;37:1798-1799.

\author{
Sandip Basu \\ Radiation Medicine Centre \\ Bhabha Atomic Research Centre \\ Tata Memorial Hospital Annexe Building \\ Jerbai Wadia Rd. \\ Parel, Mumbai, Maharashtra, India 400012 \\ E-mail:drsanb@yahoo.com
}

Published online Sep. 3, 2015.

DOI: 10.2967/jnmt.115.164046 